\title{
Die Entgrenzung von Arbeit und die Transformation raumbezogener Orientierungen - Eine Annäherung in der Region Halle/Leipzig
}

\author{
G. Montanari ${ }^{1}$, K. Wiest ${ }^{1}$, and S. Wörmer ${ }^{2}$ \\ ${ }^{1}$ Leibniz Institut für Länderkunde, Leipzig, Germany \\ ${ }^{2}$ Geographisches Institut der Universität Bonn, Bonn, Germany \\ Correspondence to: K. Wiest (k_wiest@iffl-leipzig.de)
}

Received: 22 November 2012 - Revised: 21 March 2013 - Accepted: 31 May 2013 - Published: 10 July 2013

\begin{abstract}
Zusammenfassung. Major trends in society like flexibilisation, blurring of boundaries between life spheres and subjectification of labour come along with new requirements in individual's everyday life. In the scientific debate it has in contrast hardly been discussed how these trends affect different levels of society beyond social strata like the creative class. Referring to the concepts of reflexive modernity and time-geography the focus of this article is on temporal and spatial aspects of societal change and its effects on everyday life. Based on indepth interviews and a household survey carried out in different residential areas in the region of Halle-Leipzig the paper points out how blurred borders between "work" and "life" affect individuals' space-time activities between new opportunities and new constraints. Here an inner-city neighbourhood and a community in the urban sprawl between Halle and Leipzig are under consideration to highlight different strategies to deal with weakening associations between activities, place and time emerging in different settlement structures.
\end{abstract}

\section{Einleitung}

Das Schlagwort „Entgrenzung“ beschreibt vielschichtige und grundlegende Aspekte des gesellschaftlichen Wandels der Arbeits- und Lebenswelt im Kontext der Globalisierung (z.B. Läpple et al., 2010). Im Mittelpunkt der Diskussion um eine Entgrenzung von Arbeit stehen die Auflösung eines als idealtypisch angenommenen Normalarbeitsverhältnisses und die Auswirkungen des zunehmend fließenden Übergangs von Arbeits- und Privatleben auf das individuelle Handeln im Alltag. In diesem Kontext wird im Rahmen sozialgeographischer Arbeiten vor allem die Tatsache diskutiert, dass sich verbindliche Zeitraummuster immer weiter auflösen (Pohl, 2009, 25; von Streit, 2011, 32, 37). Vertreter der wissensorientierten Ökonomien bzw. der „kreativen Klasse“ werden in diesem Zusammenhang als „Zeitpioniere“ und Vorreiter auf dem Weg zu neuen, weniger standardisierten Arbeits- und Lebensweisen betrachtet (Hörning et al., 1998; Oßenbrügge et al., 2009; von Streit, 2011; Thiel, 2008). Immer häufiger wird auf die innere Differenziertheit dieser Gruppen hingewiesen, wie es z.B. Gornostaeva und Campbell am Beispiel der vielfältigen Einkommens- und Lebensstilunterschiede innerhalb der ,creative class“ sowie der Disparitäten zwischen kreativen Eliten und einer ,creative underclass" thematisieren (2012). Angenommen wird, dass die neue Organisation der Alltagswelt veränderte Standortbedürfnisse mit sich bringt, die zu einer neuen Nachfrage nach funktionsgemischten innerstädtischen Quartieren führt (Läpple et al., 2010). Inwieweit eine Entgrenzung von Arbeit und Leben die Lebensgestaltung breiterer Gesellschaftsschichten betrifft und damit als ein gesellschaftsumfassendes Phänomen verstanden werden kann, das in unterschiedlichen raumstrukturellen Zusammenhängen gelebt wird, wurde dagegen bislang kaum thematisiert. Vor diesem Hintergrund steht im ersten Teil des vorliegenden Artikels neben einer Begriffsklärung zunächst die Raumwirksamkeit von Entgrenzungsphänomenen im Fokus. Im Anschluss wird auf der Grundlage von Haushaltsbefragungen und Tiefeninterviews der Frage nachgegangen, wie sich die Entgrenzung von Arbeit und Leben in unterschiedlichen raumstrukturellen Kontexten manifestieren kann. Dabei stehen einerseits urbane und andererseits 
zwischenstädtische Strukturen und Milieus sowie Beschäftigungsformen auch jenseits der Wissensökonomien im Mittelpunkt der Betrachtung. Die in diesem Artikel vorgestellten Ergebnisse sind Bestandteil des DFG-geförderten Kooperationsprojekts ,Wohnstandortentscheidungen in polyzentrischen Stadtregionen“. ${ }^{1}$

\section{Soziale Entgrenzung - ein gesamtgesellschaftli- ches Phänomen mit räumlichen Implikationen}

Mit dem Begriff der Entgrenzung werden spätestens seit der Entwicklung der Theorie reflexiver Modernisierung (Beck et al., 2004; Giddens, 1996) grundlegende Veränderungen sozialer Zusammenhänge thematisiert. Diese beziehen sich einerseits auf den allgemeinen Wandel gesellschaftlicher Strukturen und andererseits auf die Organisation der Arbeitswelt in einem globalen Kontext. Mit Blick auf den sozialstrukturellen Wandel werden unter anderem die Auflösung bisher gültiger und eindeutiger gesellschaftlicher Kategorien wie die Zugehörigkeit zu bestimmten Schichten, Klassen, Milieus oder Lebensstilen als gesellschaftliche Entgrenzung beschrieben: Die Tatsache, dass feste Traditionen an Gültigkeit verlieren und der Einzelne sich zunehmend selbst innerhalb der nun differenzierten gesellschaftlichen Sphären verorten muss, führt zu einem wachsenden Informationsbedarf mit dem Zwang, zwischen verschiedenen Handlungsoptionen zu wählen. Dies ist mit einer stärker ,reflexiven " Alltagsorganisation verbunden, die für das Individuum neue Grenzziehungen zwischen verschiedenen Lebensbereichen notwendig werden lässt (Beck et al., 2004). Vor diesem Hintergrund ist zu betonen, dass der Begriff der Entgrenzung nicht die Auflösung von Grenzen des alltäglichen Handelns meint, sondern vielmehr die Neuordnung und Verschiebung unterschiedlicher Lebensbereiche, die sich in veränderten Alltagsorganisationen und Mobilitätsmustern niederschlagen kann (Jurczyk et al., 2009). „Die Grenzmetapher wird dabei als heuristisches Instrument herangezogen, um für grundlegende Wandlungsdynamiken zu sensibilisieren“(Jürgens, 2009, 7).

Hinsichtlich des Wandels der Arbeitswelt sind Flexibilisierungsprozesse hervorzuheben, die Ausdruck der in den westlichen Industrienationen Ende der 1970er Jahre eintretenden Krise der fordistischen Massenproduktion sind. Wesentliches Kennzeichen postfordistischer Arbeitsbedingungen sind deregulierte Arbeitsverhältnisse und flexible, dezentralisierte Produktionsstrukturen, die zu neuen Arbeitsbiographien führen. Diese bewegen sich zunehmend ,im brei-

\footnotetext{
${ }^{1}$ Im Mittelpunkt des Projekts standen aktuelle Wanderungsmuster und die Alltagsorganisation von Bewohnern der Regionen Köln/Bonn, Östliches Ruhrgebiet und Halle/Leipzig unter dem Einfluss wachsender Mobilität und gesellschaftlicher Pluralisierungstendenzen. Es wurde von der Rheinischen Friedrich-WilhelmsUniversität Bonn, dem ILS (Institut für Landes- und Stadtentwicklungsforschung gGmbH), Dortmund und dem Leibniz-Instituts für Länderkunde (IfL) Leipzig bearbeitet.
}

ten Spannungsfeld von prekären und autonomen Arbeitsbedingungen“ (Häußermann et al., 2008, 365). Gleichzeitig wird die unter dem Stichwort der "Subjektivierung“ von Arbeit diskutierte fortschreitende Verschränkung der beruflichen, sozialen und persönlichen Lebensbereiche zu einem Charakteristikum der neuen Arbeitswelt (Jürgens, 2009, 63, 68ff.; Läpple et al., 2010, 14f.). Die beschriebenen Entwicklungen wurden bisher vor allem bei den Creative Industries mit kleinbetrieblichen Strukturen und hohen Anteilen von Selbstständigen beobachtet und dargestellt (Schiffbänker and Papouschek, 2008). Der Versuch einer Definition entgrenzter Lebensweisen offenbart jedoch, dass die in der Literatur geschilderten Kernkriterien, wie die fehlende Trennung von Arbeit und Privatleben sowie flexible Arbeitszeiten keine grundsätzlich neuen Phänomene darstellen. Für Berufsgruppen wie Lehrer und Journalisten, für Handwerksbetriebe und Unternehmer, aber auch Studierende, erscheint die aktuell diskutierte Entgrenzung von jeher als charakteristisches Merkmal der Lebensführung. Neu ist allerdings, dass die genannten Phänomene auf breitere Berufsgruppen übergreifen und weite Gesellschaftsbereiche zu durchziehen scheinen. Die raumbezogenen Konsequenzen der beschriebenen gesellschaftlichen Veränderung sind mit einem erneuten Interesse an Konzepten der Zeitgeographie und Ansätzen der Aktionsraumforschung verbunden (Ibert and Thiel, 2009; Pohl, 2010; Oßenbrügge and Vogelpohl, 2010, 69f.). Im Mittelpunkt aktionsräumlicher Ansätze, wie sie aus Torsten Hägerstrands Zeitgeographie hervorgegangen sind, stehen die Möglichkeiten und Restriktionen des raumbezogenen Handelns im Alltag, das über „Raum-Zeit-Pfade“ als physische Mobilität im Raum darstellbar wird und durch unterschiedliche Zwänge limitiert wird. Der Aktionsraum bezeichnet dabei den räumlichen Ausschnitt, in dem alltägliche Aktivitäten wie Arbeiten, sich Versorgen und Wohnen verrichtet werden. „Die äußere Reichweite der räumlichen Zielorte des Handelns bildet die Grenze des Aktionsraums“ (Werlen, 2000, 345). Im Kontext gesellschaftlicher Entgrenzungstendenzen haben sich insbesondere die coupling constraints (Koordinationszwänge) gewandelt. Während technische Entwicklungen nicht nur zu neuen Möglichkeiten der Raumüberwindung, sondern auch zu anderen zeitlichen Aktivitätsmustern führen, erhöht die Auflösung kollektiver Zeitmuster den Koordinationsaufwand im Alltag beträchtlich (Rosa, 2005, 205). Darüber hinaus sind mit der Flexibilisierung institutionalisierter Arbeits- und Öffnungszeiten die coupling constraints weiteren Veränderungen unterworfen. Generell lässt sich festhalten, dass sich potenzielle und tatsächliche Aktionsreichweiten im Kontext der Globalisierung derart gewandelt haben, ,dass sie kaum mehr als stabiles ,räumliches Muster' abgebildet werden können. ,Nähe“ und ,Ferne', unmittelbare und vermittelte Erfahrung erscheinen plötzlich in einem grundlegend neuen Verhältnis“ (Lippuner, 2003, 13).

Abgesehen von den sich wandelnden Rahmenbedingungen raumbezogenen Handelns richtet sich eine zentrale 
Kritik an aktionsräumlichen Ansätzen auf die mangelnde Berücksichtigung der gesellschaftlichen Sinngehalte raumbezogener Interaktionen (Giddens, 1996). Werlens Konzeption einer ,Sozialgeographie alltäglicher Regionalisierung“, die die gesellschaftliche Konstruktion von Raum auf der Grundlage raumrelevanter Alltagspraktiken in den Mittelpunkt ihrer Betrachtung stellt, bringt hier für die Analyse raumbezogenen Handelns wichtige Impulse. Zentral ist in diesem Zusammenhang der Begriff der räumlichen und zeitlichen Entankerung bzw. Entbettung (Giddens, 1996), der dem Entgrenzungsdiskurs weitgehend entspricht und thematisiert, dass ,,sozial-kulturelle Bedeutungen, räumliche und zeitliche Komponenten des Handelns (...) nicht mehr auf festgefügte Weise verkoppelt" sind (Werlen, 1995, 134). Da den Konstitutionsleistungen der handelnden Subjekte auf diese Weise deutlich stärkere Aufmerksamkeit geschenkt wird als den dinglichen Voraussetzungen, unter denen Handeln sich vollzieht, wurde in diesem Zusammenhang gleichzeitig kritisch auf die Tendenz einer Unterbewertung des Einflusses materieller Rahmenbedingungen hingewiesen (Scheiner, 1998).

In Abgrenzung zu einem raumdeterministischen Verständnis und ausgehend von einer gesellschaftlichen Produktion des Raumes, stehen im Rahmen der folgenden Darstellung jedoch weniger die Wirkungen räumlicher Strukturen auf individuelles Handeln im Vordergrund, Vielmehr geht es um die Frage, wie Subjekte im Kontext einer Entgrenzung von Arbeit und Leben Raumstrukturen (re)interpretieren und durch ihre Wohnstandortentscheidungen und alltäglichen Raumnutzungsmuster sozialräumliche Strukturen schaffen. Dazu werden im Folgenden zunächst wesentliche Effekte des gesellschaftlichen Wandels im Kontext des Übergangs von der Industrie- zu einer Dienstleistungs- und Wissensgesellschaft auf das raumbezogene Handeln im Alltag skizziert, die im Anschluss auf der Grundlage empirischer Beispiele veranschaulicht werden.

\section{Der Wandel der Raumnutzungsmuster}

Die raumwirksamen Effekte im Zusammenhang des Übergangs von fordistischen zu postfordistischen Regimen zeigen sich in der Reorganisation räumlicher Funktionsstrukturen und in einer Transformation der raumbezogenen Alltagspraxis (Häußermann et al., 2008, 174ff.).

Im Kontext einer Flexibilisierung von Arbeitszeiten und -orten thematisiert z.B. Pohl einen veränderten Rhythmus der Städte, der mit neuen Anforderungen an das Wohnen einhergeht, denen städtische Strukturen und Wohnquartiere in unterschiedlichem Maß entsprechen $(2009,11)$. So wird konstatiert, dass die für fordistische Gesellschaften charakteristische Funktionstrennung, die sich im Vorherrschen bestimmter räumlicher Mobilitätsmuster wie z.B. Arbeiten im Zentrum, Wohnen im Umland niederschlug, in dieser Eindeutigkeit an Bedeutung verloren hat. Gleichzeitig ist bereits an dieser Stelle zu betonen, dass raum-zeitliche Struk- turen auch unter fordistischen Bedingungen niemals durchgängig einheitlich getaktet waren. Auch eine durchgehende gesellschaftliche Entgrenzung und Flexibilisierung im Sinn des Modells der postfordistischen Stadt ist nicht zu erwarten. Die im Folgenden skizzierten Effekte einer gesellschaftlichen Entgrenzung auf die raum-zeitliche Alltagspraxis sollen vielmehr Trends aufzeigen, die für unterschiedliche soziale Gruppen und Lebenslagen eine unterschiedliche Relevanz aufweisen. Hervorzuheben sind diesbezüglich einerseits räumlich erweiterte Aktionsräume auf der regionalen sowie überregionalen Ebene, anderseits räumlich verdichtete Aktivitätsmuster auf lokaler Ebene sowie generell die Vervielfältigung und Entstandardisierung aktionsräumlicher Muster. Hinsichtlich des damit verbundenen Wandels sozialer Beziehungen schreibt Wellmann: „,communities have changed from densely-knit ,Little Boxes' (denselyknit, linking people door-to-door) to ,Glocalized' networks (sparsely-nit but with clusters, linking households both locally and globally) to ,Networked Individualism“ (sparsely-knit, linking individuals with little regard to space." $(2002,10)$.

\subsection{Die Ausdehnung aktionsräumlicher Grenzen}

Für viele Erwerbstätige haben die Flexibilisierung der Arbeitswelt und die wachsende Bedeutung der professionellen Wissensökonomien, eine deutliche Zunahme der individuellen räumlichen Mobilität zur Folge. Neue berufliche Anforderungen und die räumliche Verteilung von Expertenwissen können häufiges Reisen unumgänglich machen (Ibert and Thiel, 2009, 210). Generell werden Arbeitsorte zum Teil in immer größerer Entfernung zum Wohnort aufgesucht. Diese Tendenzen spiegeln sich in der Ausdehnung aktionsräumlicher Grenzen wider. Während die Intensität der Pendelverflechtungen mit dem engeren suburbanen Umland in deutschen Verdichtungsräumen tendenziell stagniert bzw. relativ an Bedeutung verliert, gewinnen größere Pendlerdistanzen über $30 \mathrm{~km}$ Entfernung stärker an Bedeutung - eine Entwicklung, die auch in der Region Halle/Leipzig deutlich zu erkennen ist (Pütz, 2010). Betrachtet man den voranschreitenden Ausbau der Verkehrswege und verbesserte Erreichbarkeiten, lautet eine der Kernthesen in diesem Zusammenhang, dass die räumliche Nähe gegenüber der zeitlichen Nähe an Bedeutung verliert (Schmitz, 2001, 23). Auch andere im Alltag relevante Aktivitätsorte für Konsum, Freizeit, Erholung, Bildung und soziale Kontakte können sich auf weite Bereiche einer Region ausdehnen und über diese hinausgehen (Weichhart, 2009, 5). Dass der ,vergrößerte potenzielle Aktionsraum tatsächlich genutzt wird" lässt sich anhand von Pendlerströmen nachweisen, die zunehmend häufiger zwischen Orten gleicher Hierarchiestufen und folglich in der Region tangential bzw. „kreuz und quer“ verlaufen (Schmitz, 2001, 196f.). Das Wohnquartier und die Wohnsitzkommune können dadurch im Aktivitätsnetz der Haushalte an Bedeutung verlieren, während andere Orte innerhalb und außerhalb der Region zunehmend an Bedeutung gewinnen. Darüber 
hinaus wäre auch zu diskutieren, inwieweit die Möglichkeiten soziale Kontakte durch moderne Kommunikationsmedien über große Distanzen zu unterhalten nicht zwangsläufig weniger, sondern unter Umständen sogar mehr Mobilität erzeugen können (dazu siehe Larsen et al., 2006).

\subsection{Multilokalität: mehrere Wohn- und Arbeitsorte}

Überschreitet die räumliche Distanz zwischen Wohnstandort und Arbeitsplatz die Möglichkeit des täglichen Pendelns, so wird in der Regel entweder der bisherige Wohnort aufgegeben und in Richtung des Arbeitsplatzes verlegt oder am Arbeitsort ein zweiter Wohnsitz begründet - letzteres wird als multilokales Haushaltsarrangement bezeichnet. Im Kontext der Globalisierung wird Multilokalität häufig als spezifische Ausprägung der Entankerung interpretiert (Weichhart, 2009, 1). Neben arbeitsbedingt multilokal organisierten Haushalten (zweite Wohnung am Ort der Arbeit) gewinnen im Kontext der gesellschaftlichen Pluralisierung auch familienbedingte, multilokale Haushaltsarrangements an Bedeutung (z.B. Patchworkfamilien). Die Entscheidung für Multilokalität hat in jedem Fall einen wesentlichen Einfluss auf das aktionsräumliche Handeln des Haushalts (Hilti, 2009; Weichhart, 2009, 7f.). Die Besonderheit dabei ist die Verknüpfung von zwei oder mehreren unterschiedlichen räumlichen Aktionsfeldern (Hilti, 2009, 79). In diesem Zusammenhang können sich Aktionsräume multilokaler Haushalte einerseits auf überregionaler Maßstabsebene ausdehnen, während sie sich andererseits auf Grund des hohen Organisations- und Koordinationsaufwands stärker auf zentrale Ankerpunkte auf der lokalen Ebene fokussieren.

\subsection{Die Konvergenz von Arbeits- und Lebenswelt}

Neben einer räumlichen Ausdehnung des aktionsräumlichen Handelns (Kap. 3.1) ermöglicht die moderne Kommunikationstechnik auch deutlich reduzierte räumliche Aktivitätsmuster. Die Konvergenz von Arbeits- und Wohnort mit dem Wegfall von täglichen Arbeitswegen kann ein charakteristisches Merkmal dieser Lebensführung sein. Insbesondere hochmobile Bevölkerungsgruppen, die im starken Maß beruflich überregional agieren, können im Hinblick auf ihr Privat- und Freizeitleben dann mit einer Reduzierung räumlicher Mobilität und einer Verringerung von Wegen und Distanzen reagieren (siehe auch Kap. 3.2). Möglichkeiten hierzu bieten funktionsgemischte Raumstrukturen und flexible Arbeitsformen wie z.B. das Home-Office. Auch flexible, lange Arbeitszeiten können zum Teil durch die Nähe des Wohnund Arbeitsstandorts zu anderen wichtigen alltagsrelevanten Gelegenheiten wie Geschäfte, Restaurants, Freizeitangebote kompensiert werden (von Streit, 2011, 255; Oßenbrügge and Vogelpohl, 2010). Diesen neuen, häufig besonders lokal orientierten Formen der Alltagsorganisation kommen multifunktionale Orte besonders entgegen (Hofmeister, 2006, 119; Läpple et al., 2010, 16). So wurde die Bedeutung des funktionsgemischten Quartiers, in dem alle alltagsrelevanten Bedürfnisse auf kleinem Raum organisiert werden können, am Beispiel der Beschäftigten in wissensintensiven, kreativen Branchen, bereits nachgewiesen (Pohl, 2009; von Streit, 2011). Das aktionsräumliche Handeln wird räumlich dichter und enger und kann entsprechend dem Leitbild der ,Stadt der kurzen Wege" raumwirksam werden (Pohl, 2009, 308).

Im Folgenden soll an empirischen Beispielen aus der Untersuchungsregion Halle/Leipzig gezeigt werden, wie sich die skizzierte Entgrenzung von Leben und Arbeiten im raumbezogenen Alltagshandeln breiterer Bevölkerungsgruppen widerspiegeln kann.

\section{Entgrenzung von Arbeit und Leben in der Region Halle/Leipzig}

\subsection{Deindustrialisierung und Mietermarkt}

Seit Beginn der 1990er Jahre sind in der Region Halle/Leipzig im Zuge der ökonomischen Transformation Produktionsunternehmen und Industriearbeitsplätze im überdurchschnittlichen Maß abgebaut worden. Die sozialen Folgen dieser gravierenden Deindustrialisierung spiegeln sich bis heute u.a. in einer relativen Verfestigung der Langzeitarbeitslosigkeit sowie einem relativ hohen Anteil an Leistungsempfängern und geringfügig Beschäftigten wider (SEKo Leipzig, 2009). Gleichzeitig ist die Bedeutung des Dienstleistungssektors stark gestiegen. Die Wirtschaftsstruktur der Stadt Leipzig wird heute stark durch Dienstleistungsfunktionen insbesondere im Bereich Messewesen, Medienwirtschaft/IT, Gesundheitswirtschaft sowie eine vielfältige Kreativwirtschaft geprägt (ebd.). Die Kreativwirtschaft, in der Veränderungen von Arbeitszeitstandards weit verbreitet und die Anteile der Selbstständigen und Freiberufler besonders hoch sind, repräsentiert insbesondere in Leipzig im Vergleich $\mathrm{zu}$ anderen Wirtschaftsbranchen einen wachsenden Wirtschaftszweig (Lange, 2010). In Leipzig und Umland sind im Jahr 2010 ca. 44500 Menschen im Medien- und Kreativbereich tätig gewesen (Bentele et al., 2011). Der regionale Tertiärisierungsprozess hat generell zu einer Transformation der Arbeitsverhältnisse in der Region geführt. Er manifestiert sich u.a. im deutlichen Zuwachs der Klein- und Kleinstunternehmer, dem Bedeutungsgewinn flexibler, individualisierter, aber auch prekärer Formen des Arbeitens sowie von Selbstständigen und freien Mitarbeiter (Bentele et al., 2011). Dabei können die spezifischen Strukturen des Wohnungsmarkts in der Region Halle/Leipzig zum Teil als Standortvorteil für die kreative Branche aber auch als Pullfaktor für Menschen in eher ungesicherten Arbeits- und Lebensverhältnissen gewertet werden: Wohnungsüberangebot, Leerstände und im Vergleich zu anderen deutschen Großstädten günstige Mietpreise, erleichtern Existenzgründungen, eröffnen aber auch gröBere Spielräume, um neuen Anforderungen an die Alltagsorganisation auch in Bezug auf das Wohnen gerecht zu werden. Wohnstandortentscheidungen - so die Hypothese - können 
eher im Hinblick auf Flexibilisierung und Entgrenzung optimiert werden.

\subsection{Untersuchungsdesign}

Um einerseits entgrenzte Lebensweisen sowohl quantitativ als auch qualitativ zu erfassen und sich andererseits den besonderen Merkmalen der räumlichen Orientierung im Kontext von Flexibilisierung und Entgrenzung anzunähern, wird im Folgenden auf die Ergebnisse standardisierter Haushaltsbefragungen und qualitativer Interviews zurückgegriffen, die in fünf Wohnquartieren der Region Halle/Leipzig durchgeführt wurden. Im Rahmen der Haushaltsbefragungen wurden Ausprägungen entgrenzter Lebensformen auf der Grundlage von Kriterien, die mit einer fehlenden zeitlichen und räumlichen Fixierung von Arbeit und Leben im Zusammenhang stehen, identifiziert. Als empirische Indikatoren für eine zeitliche Entgrenzung können u.a. sehr unregelmäßige Arbeitszeiten, häufige Überstunden sowie Schicht- und Wochenendarbeit gesehen werden. Hinweise auf eine raumbezogene Entgrenzung lassen sich aus überdurchschnittlich weiten Pendeldistanzen, einer fehlenden räumlichen Trennung zwischen unterschiedlichen Funktionsbereichen (z.B. Home-Office), mehreren Arbeits- und Wohnorten (Multilokalität) sowie mehreren Beschäftigungsverhältnissen (zusätzliche Tätigkeiten) ablesen.

Darüber hinaus wurden im Rahmen der Erhebungen die wichtigsten alltagsrelevanten Aktivitätsorte der Haushaltsmitglieder erfasst (Arbeitsort, wichtige Versorgungsstandorte und Zielorte der Freizeitgestaltung). Diese Angaben lassen zwar keine detaillierte Aufzeichnung von Raum-ZeitPfaden zu, ermöglichen jedoch eine Annäherung an die Raumnutzungsmuster der Bewohner in unterschiedlichen raumstrukturellen Zusammenhängen. Da die Fokussierung auf die physische Mobilität der Akteure im Raum Sinngehalte von Interaktionen und die Bedeutung von Orten für individuelles Handeln ausgeklammert lässt, zielten die Tiefeninterviews mit Bewohnern vor allem auf die Frage ab, wie sich die Flexibilisierung der Arbeitswelt und der Bedeutungsverlust von räumlichen und zeitlichen Bindungen in individuellen Handlungsstrategien niederschlagen und subjektiv erlebt werden können. Die Auswahl der Gesprächspartner sollte sich hier nicht auf die sogenannte ,kreative Klasse“ beschränken. Ziel war es vielmehr, Erkenntnisse darüber zu gewinnen, wie sich die angenommene Entgrenzung von Arbeit und Leben auf das raumbezogene Handeln im Alltag ganz unterschiedlicher Bevölkerungsgruppen auswirkt. Insgesamt wurden 20 Gespräche mit Quartiersbewohnern geführt, deren Lebensweise deutliche Hinweise auf eine entgrenzte Lebensweise erkennen ließ.

\subsection{Muster räumlicher Orientierung in urbanen und zwischenstädtischen Kontexten}

Um Zusammenhänge zwischen räumlichen Handlungsmustern und unterschiedlichen raumstrukturellen Kontexten herauszuarbeiten, werden im Folgenden ein urbanes, innerstädtisches Quartier und ein zwischenstädtischer Wohnstandort näher betrachtet. Urbane Quartiere werden häufig mit dem Leitbild der kompakten Stadt assoziiert, in dem Dichte und kleinteilige Nutzungsmischung vorherrschen. Demgegenüber setzt sich der Begriff der Zwischenstadt im Sinn eines Gegendiskurses zur kompakten Stadt mit neuartigen, diffusen Übergangszonen zwischen urbanen und ländlichen Zonen im Kontext einer Entgrenzung der Stadt auseinander. Die Südvorstadt, ein Leipziger Cityrandgebiet und die Ortsteile Gröbers/Großkugel der Gemeinde Kabelsketal, auf der Siedlungsachse Halle/Leipzig gelegen, können jeweils als $\mathrm{Mu}-$ sterbeispiel für diese beiden Raumstrukturtypen betrachtet werden. Ein wichtiges Kriterium für die Auswahl der beiden Quartiere war neben ihrer Lage innerhalb der Region das Versorgungsangebot mit Gütern und Dienstleistungen sowie die ÖPNV-Erreichbarkeit, da beide Merkmale einen grundlegenden Einfluss auf das aktionsräumliche Verhalten im Alltag ausüben.

\subsubsection{Leipzigs Südvorstadt - Ein urbanes Szeneviertel}

Die Südvorstadt repräsentiert ein innenstadtnahes, gründerzeitliches Wohnquartier, das über eine sehr hohe Versorgungs- und Erschließungsqualität verfügt: Die Leipziger Innenstadt ist mit dem öffentlichen Verkehr in ca. 510 Minuten erreichbar. An der zentralen Verkehrsachse des Quartiers reihen sich zahlreiche Geschäfte, Dienstleistungsund Gastronomiebetriebe. Nicht zuletzt bedingt durch die Nähe zu Standorten der Medien- und Kreativwirtschaft (Mediacity, MDR) sowie Hochschulen und Forschungseinrichtungen ist die Bevölkerungszusammensetzung des Stadtteils stark geprägt durch Studenten, junge Berufseinsteiger aus dem qualifizierten Dienstleistungssektor und dem akademischen Bereich sowie der Kulturszene (Gerdes et al., 2009, 183). Mit diversen Gaststätten, Kneipen und Straßencafés hat das Gebiet den Ruf des bunt gemischten Szeneviertels. Die Südvorstadt ist somit durch Strukturen gekennzeichnet, die in der Literatur typischerweise mit der Auflösung fordistischer Raum-Zeit-Muster in Verbindung gebracht bzw. als postfordistisch bezeichnet werden (z.B. Läpple et al., 2010).

\subsubsection{Gröbers/Großkugel - Zwischenstädtische Strukturen auf der Achse Halle/Leipzig}

Die Ortsteile Gröbers und Großkugel der Gemeinde Kabelsketal repräsentieren einen zwischenstädtischen Wohnstandort, der sowohl durch alte Dorfkerne als auch umfangreiche und unterschiedlich strukturierte Neubaugebiete geprägt ist, die im Zuge von Wachstumserwartungen und steuerlichen Sonderabschreibungen nach 1990 neu und relativ ungeplant 
Tabelle 1. Indikatoren für eine räumlich bzw. zeitlich entgrenzte Lebensweise nach Wohnstandorten.

\begin{tabular}{llll}
\hline & Leipzig Südvorstadt & Gröbers/Großkugel & $\begin{array}{l}\text { Gesamtstichprobe } \\
\text { Halle/Leipzig }\end{array}$ \\
\hline Überstunden $>50 \mathrm{~h}$ & $6,7 \%(n=180)$ & $8,0 \%(n=100)$ & $6,9 \%(n=750)$ \\
Schichtarbeit & $9,8 \%(n=183)$ & $22,9 \%(n=105)$ & $12,1 \%(n=771)$ \\
multilokale Lebensweise & $16,0 \%(n=176)$ & $6,8 \%(n=103)$ & $7,1 \%(n=771)$ \\
(teilweise) Home-Office & $16,4 \%(n=183)$ & $2,9 \%(n=105)$ & $9,1 \%(n=771)$ \\
zusätzliche Tätigkeit & $14,9 \%(n=175)$ & $13,5 \%(n=104)$ & $11,0 \%(n=743)$ \\
Fernpendler (einfacher Arbeitsweg $>50 \mathrm{~km})$ & $7,1 \%(n=170)$ & $8,2 \%(n=98)$ & $6,7 \%(n=722)$ \\
\hline Sozioökonomische Merkmale der Befragten mit entgrenzter Lebensweise & \\
\hline Anteil (Fach-)Hochschulabschluss & $69 \%(n=74)$ & $39 \%(n=26)$ & $59,5 \%(n=764)$ \\
Anteil Einpersonenhaushalte & $37 \%(n=74)$ & $15 \%(n=26)$ & $14,9 \%(n=768)$ \\
\hline
\end{tabular}

(Die Werte beziehen sich jeweils auf die angestellt und selbstständig Erwerbstätigen.) Quelle: eigene Erhebung

entwickelt wurden. Die sehr günstige PKW- und ÖPNVErreichbarkeit der Städte Halle und Leipzig hat die Kommune zu einem bevorzugten Standort der Logistikbranche gemacht. Da die Bevölkerungsentwicklung Gröbers und Großkugels weit hinter den ursprünglichen Erwartungen zurückgeblieben ist, stellt sich auch die Versorgung mit Gütern und Dienstleitungen nach wie vor als unzureichend dar. Für die Zusammensetzung der Bevölkerung an diesem Standort ist eine Mischung aus alteingesessenen und neu zugezogenen Bewohnern charakteristisch. Eine starke Fluktuation sowie eher unterdurchschnittliche Bildungsabschlüsse in einzelnen Ortsteilen deuten auf sozial selektive Mobilität hin.

\subsection{Entgrenzte Lebensweisen und Aktivitätsorte zwischen neuen Handlungsoptionen und neuen Zwängen}

Die Gegenüberstellung der beiden Untersuchungsstandorte macht deutlich, dass die ausgewählten Indikatoren einer zeitlichen und räumlichen Entgrenzung in den beiden Quartieren in unterschiedlichem $\mathrm{Ma} ß$ auf die Lebenssituation der Bewohner zutreffen. So ist in der Südvorstadt eine vergleichsweise starke Ausprägung des Anteils multilokal orientierter Personen erkennbar (Tabelle 1). Auch das Home-Office, als Paradebeispiel für die Überwindung der raum-zeitlichen Trennung von Erwerbsarbeit und Privatleben ist hier überdurchschnittlich stark vertreten. In Gröbers/Großkugel hat dagegen vor allem die Schichtarbeit große Bedeutung. Darüber hinaus sind auch das Fernpendeln und das Ausüben einer zusätzlichen Tätigkeit etwas stärker verbreitet. Arbeit im Home-Office ist mit $3 \%$ der angestellt und selbstständig Erwerbstätigen dagegen kaum von Bedeutung. Grundsätzlich können Multilokalität und die Arbeit im Home-Office für sehr unterschiedliche Lebensformen und für verschiedenste berufliche Tätigkeiten charakteristisch sein. Das Ausüben zusätzlicher Tätigkeiten, aber auch Fernpendeln verweisen dagegen eher auf Strategien, die im Zusammenhang mit einem restriktiven Teilarbeitsmarkt bzw. prekären Lebensla- gen stehen. Die Unterschiede in der sozialen Zusammensetzung zwischen den Befragten in der Südvorstadt und Gröbers/Großkugel, die Merkmale einer entgrenzten Lebensweise zeigen, stärken diese Vermutung. Sie deuten hier auf unterschiedliche sozioökonomische Handlungsspielräume der Bewohner sowohl auf dem Arbeits- als auch dem Wohnungsmarkt an den beiden Standorten hin (Tabelle 1).

Um sich der Frage anzunähern, inwieweit sich die Entgrenzung von Arbeit und Leben in spezifischen Raumnutzungsmustern niederschlägt, werden im Folgenden Zielorte zentraler Alltagsaktivitäten für jene Personen abgebildet, für die entweder eine berufliche Tätigkeit im Home-Office, das Ausüben einer zusätzlichen Tätigkeit, Fernpendeln (einfacher Arbeitsweg $>50 \mathrm{~km}$ ) oder eine multilokale Lebensweise zutreffend sind. Die in Abb. 1a dargestellte räumliche Anordnung der Arbeitsorte veranschaulicht unterschiedliche Aspekte einer Entgrenzung des Erwerbslebens. So fällt in der Leipziger Südvorstadt - abgesehen von der relativen Bedeutung des Home-Office - auf, dass häufig in Wohnortnähe, im Bereich der Leipziger Innenstadt sowie in südlich angrenzenden Stadtteilen gearbeitet wird. Auf der anderen Seite sind die Arbeitsorte zum Teil in sehr großer Distanz, z.B. in den alten Bundesländern lokalisiert und weisen auf multilokale bzw. hochmobile Lebensformen hin. Dieser Befund könnte dahin gehend näher untersucht werden, inwieweit vor allem die beruflich hochmobilen Bevölkerungsgruppen das Bedürfnis haben sich privat verstärkt am Wohnort im Sinn einer „Homebase“ zu verankern, wie es Ibert et al. vor dem Hintergrund des besonders unsicheren Arbeitsmarktes von Musicaldarstellern beispielhaft aufzeigen (2012). Hinweise hierzu finden sich auch in unseren Ausführungen (s.u.).

In Gröbers/Großkugel sind die Arbeitsorte der Untersuchungsgruppe dagegen eher flächig und dispers in der Region verteilt und befinden sich vor allem außerhalb des eigenen Quartiers. Dieses Muster spiegelt zum Teil die Gegebenheiten der Siedlungsstrukturen und der Arbeitsplatzverteilung innerhalb der Region wider. Aber auch die relative Bedeutung des (Fern)Pendelns in Gröbers/Großkugel wird 


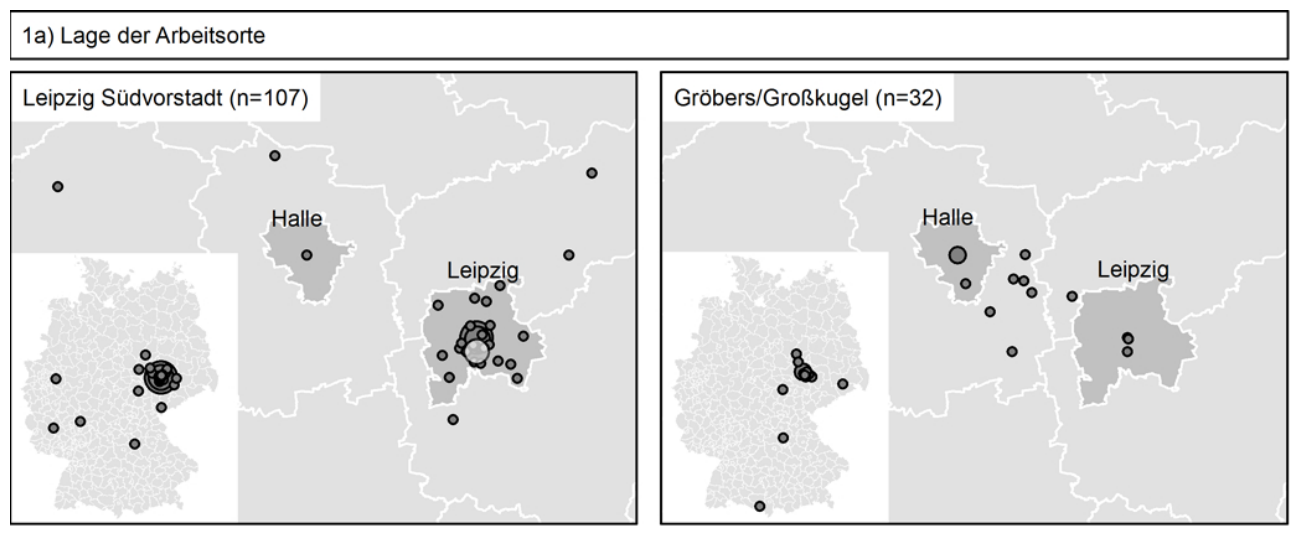

1b) Standorte „Lebensmittel einkaufen“

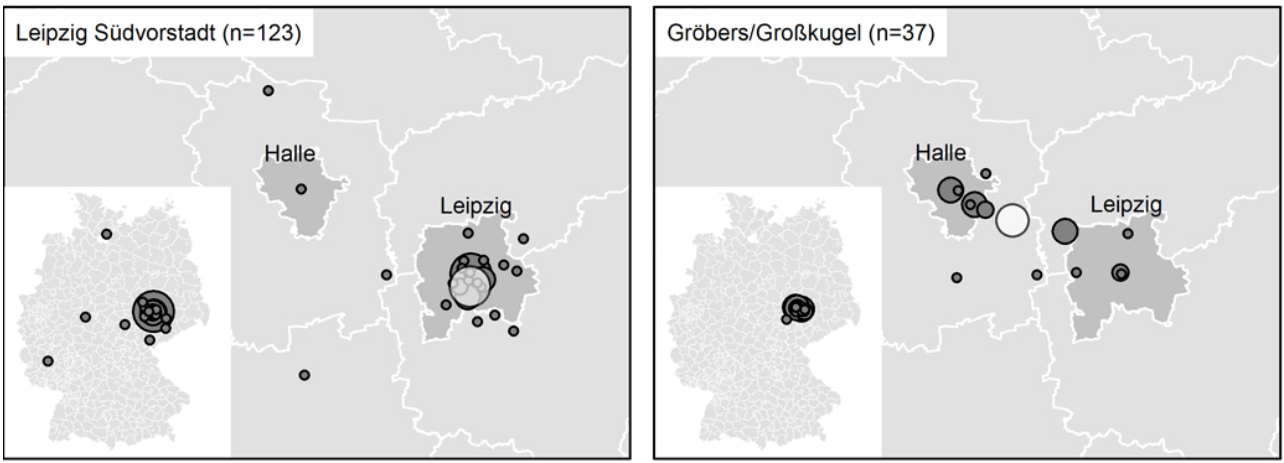

1c) Standorte „in ein Café oder eine Kneipe gehen"
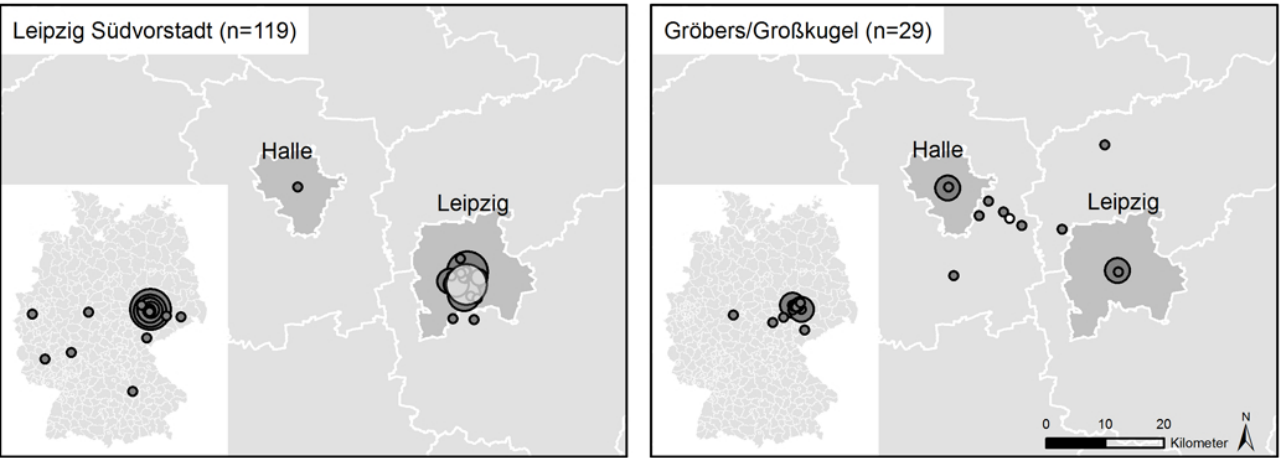

- 1 bis 5 O 6 bis 10

11 bis 20

21 bis $50 \bigcirc$ über 50

eigenes Quartier

Abbildung 1. (Räumlich) Entgrenzte Lebensweisen und Aktivitätsort (Quelle: eigene Darstellung, Grundlagenkarte: OpenStreetMap und Mitwirkende, cc-by-sa).

erkennbar. Teilweise kommen an diesem Standort die im Zuge der Entgrenzung von Arbeit und Leben entstehenden neuen Handlungszwänge, als vom Arbeitgeber bzw. dem Arbeitsmarkt gesetzte Einschränkungen und erhöhte Anforderungen an die Flexibilität stärker zum Tragen. So sind kurzfristige Arbeitsverhältnisse mit wechselnden Einsatzorten als wesentlicher Aspekt der Entgrenzung - in Abb. 1a zwar nicht darstellbar. Das folgende Zitat am Beispiel einer Fernpendlerin mit zeitlich befristetem Arbeitsvertrag veranschaulicht in diesem Zusammenhang jedoch, wie die Entstandar- disierung und Flexibilisierung der Arbeitswelt vor allem als fehlende Planungssicherheit erlebt werden. Die Wahl eines möglichst verkehrsgünstigen Wohnstandorts zwischen Halle und Leipzig kann als Versuch interpretiert werden, sich mit dieser Ungewissheit und einer Entstandardisierung der Arbeitswege zu arrangieren. Da in diesem Fallbeispiel unklar ist, ob sich der finanzielle und zeitliche Aufwand des Pendelns auch langfristig lohnen wird, werden die weiten Arbeitswege als umso größere Belastung wahrgenommen: 
Man ist komplett gefrustet, erst mal weil der Weg so lang ist, dann weil ich die Tanknadel sehe, dann weil ich weiß, ok, es ist eh nur für ein halbes Jahr, was sollst du dich hier reinhängen, ähm danach wirste eh woanders hingesetzt und die Bank [in der sie angestellt ist] ist groß, es geht bis Merseburg, bis Querfurt hinter, ich weiß nicht wo die mich da hinsetzen. (Frau S., 30 Jahre, Bankangestellte, Gröbers/Großkugel)

Um raumbezogene Orientierungen im Alltag abzubilden, wurden die Befragten gebeten, neben den Arbeitsorten auch verschiedene Alltagsaktivitäten, wie Lebensmitteleinkäufe und Freizeitbeschäftigungen zu lokalisieren. Abbildung 1b und $\mathrm{c}$ zeigen beispielhaft die Zielorte für Lebensmitteleinkäufe und Café- bzw. Kneipenbesuche. Sowohl hinsichtlich des täglichen Einkaufs als auch des Café- bzw. Kneipenbesuchs wird bei den Befragten in der Südvorstadt eine sehr ausgeprägte Quartiersorientierung deutlich. Die infrastrukturelle Dichte und das vielfältige gastronomische Angebot in der Leipziger Südvorstadt werden dabei von den Befragten ganz bewusst als Standortvorteil wahrgenommen und gezielt genutzt, um die zum Teil hohen Arbeitszeitbelastungen zu kompensieren. Die folgende Aussage eines Speditionsangestellten, dessen Arbeitsalltag durch sehr unregelmäßige und überlange Arbeitszeiten geprägt ist, illustriert darüber hinaus, dass das vielfältige infrastrukturelle Angebot im Quartier die Nachteile eines weiten Arbeitswegs bei weitem zu überwiegen scheint. So wird ein alternativer Wohnstandort, der sich in größerer Nähe zum Arbeitsplatz befindet, in diesem Beispiel gar nicht erst in Erwägung gezogen:

Dadurch, dass auch arbeitstechnisch die Zeit knapp bemessen ist, was Freizeit anbetrifft, da bietet sich das halt an, in dem Stadtteil zu wohnen, wo du viele Sachen auf einem Haufen hast, um da die Zeit wieder, sozusagen, reinzuholen... und dann ist es natürlich hier auch so, dass man immer, übertrieben gesagt, das bekommt, was man grad möchte... (Herr L., 27 Jahre, Speditionsangestellter, Südvorstadt)

Das Fallbeispiel veranschaulicht, wie sich die Optimierung von Versorgungswegen durch eine bewusste Wohnstandortentscheidung, die flexiblen Öffnungszeiten der vor Ort vorhandenen Einrichtungen und die individuelle Alltagsorganisation zu einer spezifischen Lebensweise nach dem Motto ,alles zu gleicher Zeit am gleichen Ort“" (vgl. Hofmeister, 2006; Oßenbrügge und Vogelpohl, 2010, 68) verdichten können. Es wird deutlich, dass trotz der vorhandenen Möglichkeiten der Raumüberwindung - oft nicht zuletzt begünstigt durch eine bewusst reflexive Lebensführung - neue Standortanforderungen entstehen, die mit neuen räumlichen Bindungen verknüpft sein können. Diese sind durchaus im Sinn einer Rückbettung bzw. Wiederverankerung des sozialen Lebens an lokale raum-zeitliche Gegebenheiten zu verstehen (Giddens, 1996). Auf der anderen Seite sind weite und scheinbar regellose Aktivitätsräume zu beobachten, die unter dem Aspekt der zunehmenden Loslösung sozialer Beziehungen aus ortsgebundenen Interaktionszusammenhängen betrachtet werden können. Bereits die Darstellung der Versorgungszielorte in den beiden Untersuchungsquartieren deutet hier auf sehr unterschiedliche Orientierungsmuster und raumbezogene Bindungen hin: Im Unterschied zu der relativ starken Ausrichtung der Aktivitäten auf den Nahbereich in der Südvorstadt werden von der Untersuchungsgruppe in Gröbers/Großkugel viele Standorte in der Region, insbesondere auf der Siedlungsachse Halle-Leipzig, angefahren. Neben den beiden Stadtzentren sind dies vor allem Einkaufsmärkte auf der „Grünen Wiese“. Betrachtet man die Destinationen eines Café- oder Kneipenbesuchs, bilden die Oberzentren Leipzig und Halle dominante Anlaufpunkte.

Dass sich die räumlichen Aktivitätsmuster auch aus der Sicht der Befragten an den beiden Standorten sehr unterschiedlich darstellen, unterstreicht darüber hinaus der Grad der Zustimmung zu den beiden Statements „Ich verbringe viel Zeit im Quartier“ bzw. „Ich bin viel in der Region unterwegs". Die auch subjektiv wahrgenommene lokale Orientierung am urbanen Wohnstandort und die regionale Orientierung am zwischenstädtischen Standort wird in Abb. 2 deutlich. Grundsätzlich legen die Untersuchungsergebnisse den Schluss nahe, dass die unterschiedlichen Aktivitätsmuster an den beiden Standorten in erster Linie auf die vorhandenen räumlichen Opportunitäten zurückgeführt werden müssen. Die Entscheidung an einem der beiden Wohnstandorte zu leben, ist jedoch das Ergebnis aus (tatsächlichen und wahrgenommenen) Handlungsmöglichkeiten und den spezifischen, oft sehr individuellen Vorstellungen über die Lebensführung und Alltagsorganisation zu sehen. Diesen Aspekt veranschaulicht die Bewertung von Wohnstandortkriterien differenziert nach Befragten, die unterschiedliche Kennzeichen einer entgrenzten Lebensführung aufweisen sowie der Vergleich zum Gesamtdurchschnitt der von uns insgesamt befragten Haushalte (Abb. 3). Während zum Beispiel die Befragten mit Home-Office die Nähe zu Freunden und Verwandten, das Image sowie Kultur- und Freizeitangebote vergleichsweise häufig als wichtig bzw. sehr wichtig beurteilen, kommt bei Schichtarbeitern und Personen mit zusätzlichen Tätigkeiten der Kostenfaktor besonders stark zum Tragen. Bei Befragten, die sehr viele Überstunden machen (Anbindung an das überörtliche Verkehrsnetz) sowie bei Schichtarbeitern (PKW-Stellplätze) ist eine stärkere PKW-Orientierung erkennbar; Personen mit zusätzlichen Tätigkeiten und im Home-Office erscheinen wiederum eher ÖPNV-orientiert. Für Schichtarbeiter ist das ruhige Wohnumfeld und die Nähe zum Arbeitsort bedeutend, für Befragte mit Zweitwohnung oder vielen Überstunden stehen das kindergerechte Wohnumfeld und die Kinderbetreuung eher im Vordergrund. Befragte im Home-Office, das häufig als Arbeitsform betrachtet wird um Beruf und Familie besser zu vereinbaren, gewichten dagegen die Kinderbetreuung auffällig gering. Als Erklärung kann diesbezüglich herangezogen 


\section{Statement: \\ "Ich verbringe viel Zeit im Quartier"}

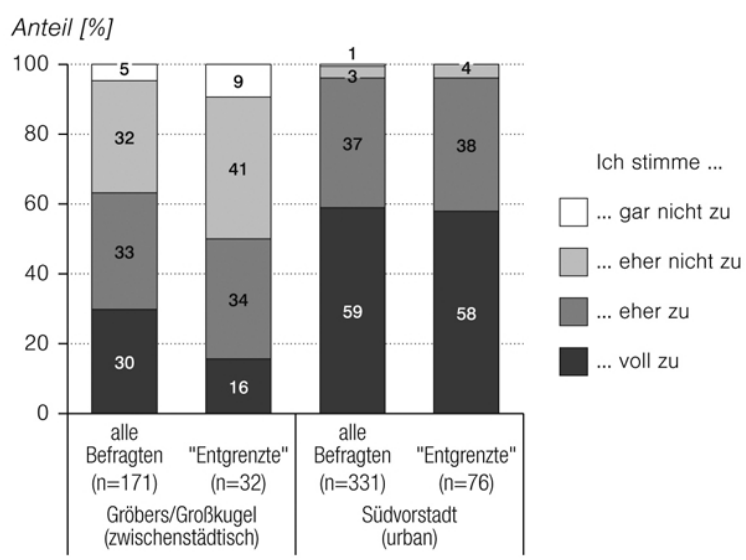

\section{Statement: \\ "Ich bin viel in der Region unterwegs"}

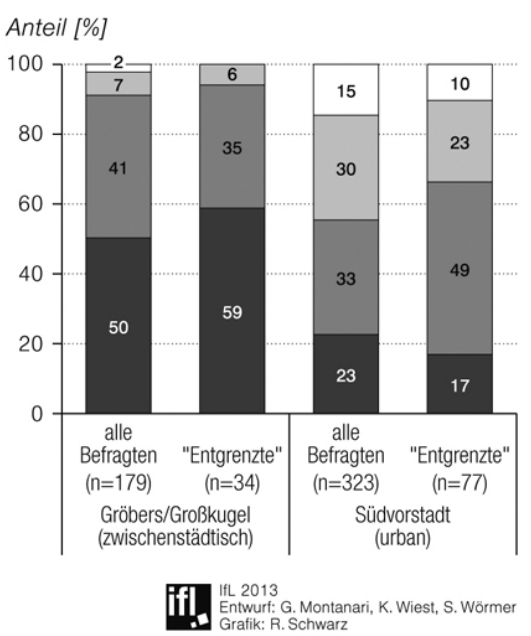

Abbildung 2. Wahrnehmung des eigenen Aktionsraums - Bewertung von Statements.

werden, dass Befragte im Home-Office einerseits die Kinderbetreuung zum Teil selbst übernehmen, andererseits hier sogar zum Teil weniger Kinder im Haushalt leben. Diese Ergebnisse deuten an, dass unterschiedliche Formen einer Entgrenzung mit unterschiedlichen sozialen Schichten und spezifischen Lebensstilen korrespondieren, denen Wohnstandorte im unterschiedlichen Maß entsprechen. Dass Wohnstandortentscheidungen letztendlich immer auch für eine bestimmte Form der Lebensführung und der persönlichen Prioritätensetzung stehen, verdeutlicht das Gespräch mit einem Grafikdesigner in der Südvorstadt:

Aber ich glaub, ich bin auch eine der Ausnahmen, weil ich glaub, echt viele (...) Wohnstandortentscheidungen, sind ganz viel nach Arbeit orientiert, ... bist ja jetzt sozusagen auch hergezogen wegen der Arbeit. (...) War für mich zum Beispiel noch nie ne Option. Da such ich lieber länger. (Herr A., 28 Jahre, Grafikdesigner, Südvorstadt)

So kann auch das Leben in einer Wohnsitzkommune mit kleinräumig sehr ungünstiger Versorgungslage und dem damit verbundenen Zwang zur Ausdehnung des Aktionsraums wie in Gröbers/Großkugel als Privileg gedeutet werden, wenn eine PKW-Verfügbarkeit die Erreichbarkeit anderer Standorte möglich macht:

Mal in Leipzig (...) oder in Halle, (...), also auch bei den Ärzten ist mir das vollkommen egal. Ich habe halt das Privileg, dass ich in der Mitte wohne und sagen kann, ok, ich gehe nach Halle oder ich gehe nach Leipzig, das ist mir vollkommen egal. (Frau S., 30 Jahre, Bankangestellte, Gröbers/Großkugel)
Entgrenzung zeigt sich in diesem Beispiel darin, dass es keine festen Zielorte gibt und immer wieder neu abgewägt wird, welche Angebote angesteuert werden. Der spezifische Ort erfährt dabei in Bezug auf die Handlungsweise der Akteurin eine gewisse Beliebigkeit - ein Befund der sich unter dem Aspekt einer Entankerung bzw. Entbettung interpretieren lässt. Das Leben am zwischenstädtischen Standort wird dabei positiv, als Leben in „,der Mitte“ empfunden, das Wahlfreiheit zwischen vielen Optionen eröffnet.

Generell zeigten sich einige Gesprächspartner der Untersuchungsgruppe als wenig distanzsensibel, auch wenn die Mobilität mit einem erheblichen zeitlichen und finanziellen Aufwand verbunden ist. Andere stellen dagegen die Reduzierung von Wegen in den Mittelpunkt ihrer Alltagsorganisation - das ,Zeitsparen“ wird dann zur handlungsleitenden Maxime. Häufig wird eine Art „Mobilitätskonto“ geführt: Fallen wie im folgenden Beispiel weite Arbeitswege an, wird versucht lange Freizeitwege mit der Wahl des Wohnstandorts zu vermeiden oder aber Freizeitaktivitäten werden gänzlich reduziert:

Ich geh immer nur einkaufen. Man hat nach dem Pendeln auch keine Lust mehr noch irgendwas zu machen, beziehungsweise jetzt wo die Kleine da ist, kann ich eh abends nichts mehr machen. (Frau S., Bankangestellte, Großkugel)

Generell führen wachsende Arbeitszeitbelastungen und die Zwänge der Erreichbarkeit zu einem subjektiv sehr engen Zeitbudget, so dass versucht wird Wege und somit Zeit einzusparen. Individuelle Zeitverfügbarkeit und Zeitsouveränität werden so zu zentralen Merkmalen der Lebensqualität. Gleichzeitig machen die Untersuchungsergebnisse deutlich, 


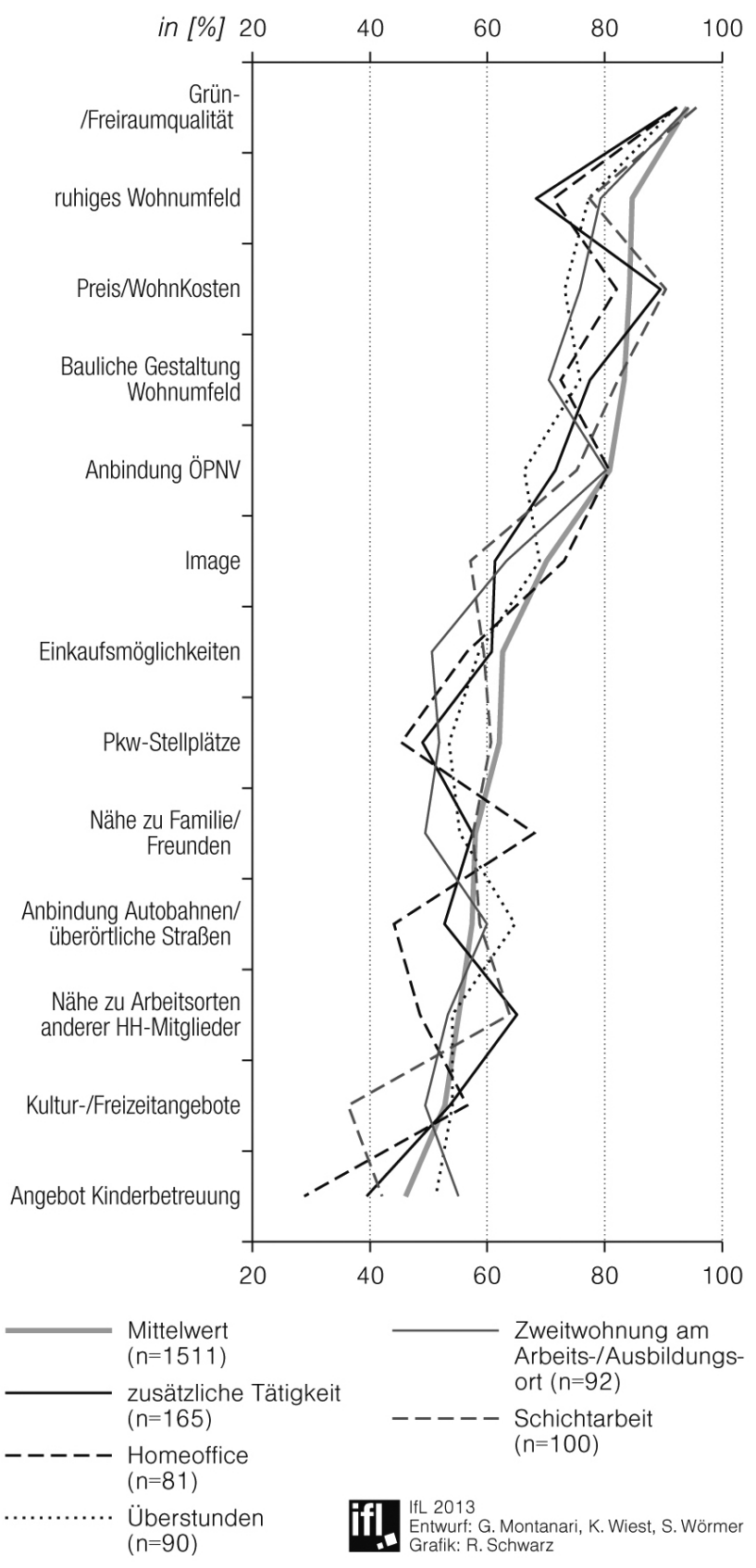

Abbildung 3. Die Bewertung mit ,wichtig“ bzw. „sehr wichtig“ von Wohnstandortkriterien durch Personen mit entgrenzter Lebensweise (Quelle: eigene Darstellung).

dass die diskutierten Auswirkungen einer Entgrenzung von Arbeit und Leben so komplex und heterogen in die individuelle Lebensführungen integriert sind, dass sie sich über die rein quantitative Beschreibung von Aktivitätsorten oder die physischen Bewegungen von Individuen im Raum nur grob identifizieren lassen.

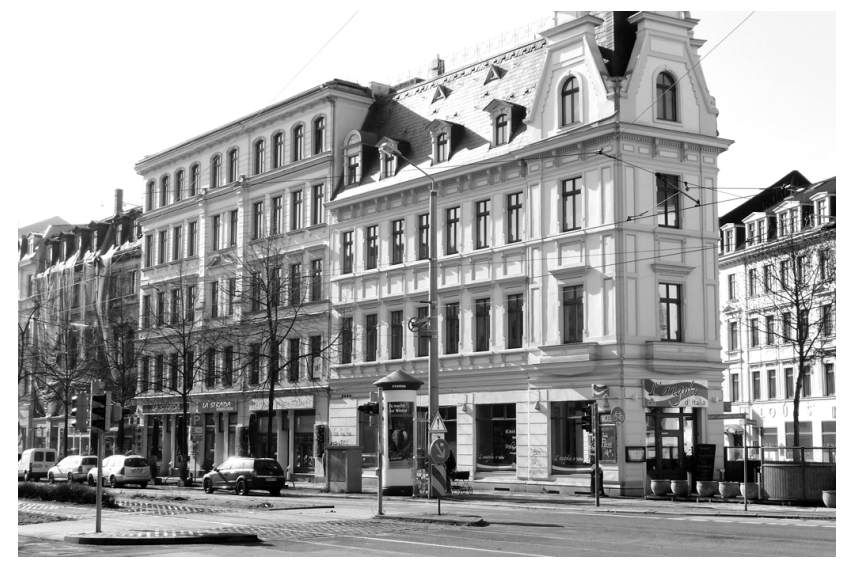

Abbildung 4. Urbaner Wohnstandort: Leipzig-Südvorstadt (Foto: S. Haunstein 2011).

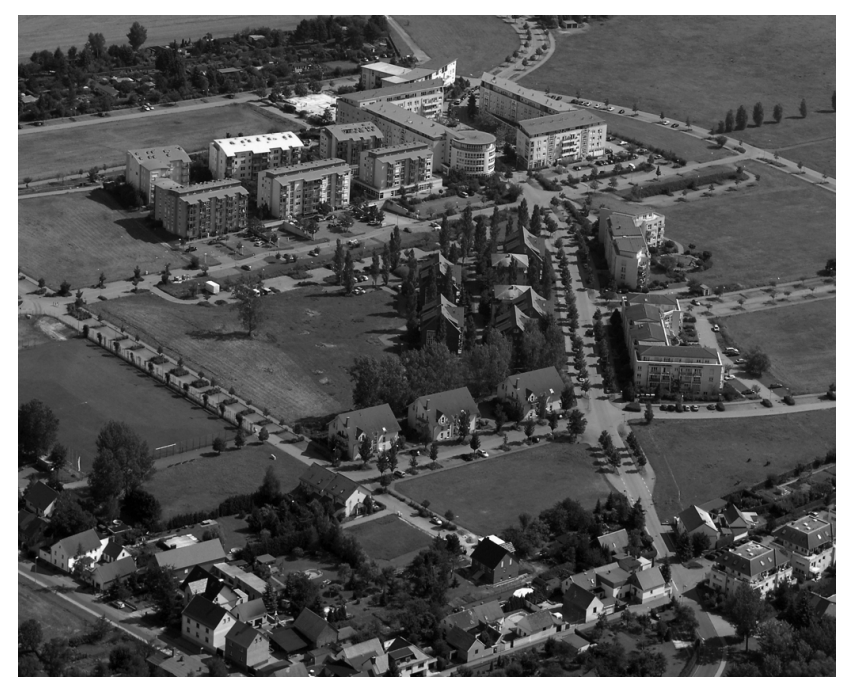

Abbildung 5. „Zwischenstädtischer“ Wohnstandort Gröbers/ Großkugel (Foto: HVB Immobilien AG/Euroluftbild Robert Grahn).

\section{Resümee}

Die Darstellung der Zielorte der Befragten lässt am zwischenstädtischen Standort generell räumlich ausgedehntere Aktivitätsmuster mit mittleren Distanzen auf der regionalen Ebene erkennen, während am urbanen Standort eine überwiegend kleinräumige und stark wohnumfeldbezogene Orientierung offensichtlich ist. Gleichzeitig ist auch der Anteil der Befragten mit multilokaler Lebensweise und Aktionsräumen, die sich auf die überregionale Maßstabsebene ausdehnen im urbanen Untersuchungsquartier überdurchschnittlich hoch. Die unterschiedlichen Aktivitätsmuster an den beiden Standorten lassen sich zum Teil durch die Gegebenheiten der Siedlungsstruktur sowie dem Angebot an Arbeitsplätzen und Versorgungseinrichtungen erklären. Insbesondere in diesem Zusammenhang ist jedoch auf die Erzeugung von Raumstrukturen als Resultat gesellschaftlicher 
Entgrenzungstendenzen hinzuweisen: Die dargestellten Alltagspraktiken der Untersuchungsgruppe an beiden Standorten müssen im Kontext von individuellen Wohnstandortentscheidungen, Strategien der Alltagsorganisation und unterschiedlichen Lebenssituationen der Bewohner interpretiert werden. Funktionsgemischte Quartiere mit räumlich konzentrierten Möglichkeitsstrukturen erscheinen vor dem Hintergrund subjektiv enger Zeitbudgets vielfach als Gewinner. Dabei können entgrenzte Lebensweisen am urbanen Standort vor allem im Kontext einer Wiederverankerung des sozialen Lebens auf lokaler Ebene interpretiert werden. Am zwischenstädtischen Standort werden dagegen eher die Konsequenzen einer Loslösung sozialer Beziehungen aus ortsgebundenen Interaktionszusammenhängen im Sinn der Entbettung offensichtlich. Das Beispiel Gröbers/Großkugel lässt erkennen, dass eingeschränkte Möglichkeitsstrukturen, fehlende Teilhabe am sozialen, kulturellen und politischen Leben und Entgrenzung im engen Wechselverhältnis zu einander stehen können. Auf der Basis quantitativer Daten konnte gezeigt werden, dass unterschiedliche Indikatoren für eine entgrenzte Lebensweise im engen Zusammenhang mit sozialen Strukturen und spezifischen Lebensstilen stehen. Diese sind allerdings aufgrund ihrer Vielschichtigkeit kaum eindeutig zu erfassen. Im Rahmen der Tiefeninterviews wurde dagegen deutlich, wie Entgrenzungstendenzen den gesellschaftlichen Alltag durchdringen und neue Formen sozialer Ungleichheit produzieren können. In Abhängigkeit von den jeweiligen Wahlmöglichkeiten auf dem Wohn- und Arbeitsmärkten kann Flexibilisierung und Entgrenzung eher positiv in Form größerer Zeitsouveränität gelebt werden oder aber in wachsenden Unsicherheiten, ständiger Zeitknappheit und hohen Mobilitätsbelastungen zum Ausdruck kommen. Vor diesem Hintergrund sollten unsere Ausführungen die Aufmerksamkeit insbesondere auf Entgrenzungsphänome lenken, die sich abseits der Wissensökonomien und der sogenannten „kreativen Klasse“ vollziehen, die vielmehr prekäre Lebenszusammenhänge betreffen und zeigen, wie sich eine Entgrenzung - umfassender als bisher begriffen - von Leben und Arbeit auch an Standorten manifestieren kann, die im Kontext der Reurbanisierungsdebatten meist vergessen werden.

Edited by: B. Korf

Reviewed by: two anonymous referees

\section{Literatur}

Beck, U., Giddens, A., und Lash, S.: Reflexive Modernisierung Eine Kontroverse, Suhrkamp Verlag, Frankfurt am Main, 2004.

Bentele, G., Liebert, T., Fechner, R., und Nießen, C.: Medien und Kreativstandort Leipzig, 2011.

Gerdes, J., Hennig, A., and Rieger, K.: Leipziger Lebenslagenreport 2009, Leipzig, 2009.

Giddens, A.: Konsequenzen der Moderne, Suhrkamp Verlag, Frankfurt am Main, 1996.

Gornostaeva, G. und Campbell, N.: The creative underclass and the production of place, J. Urban Aff., 34, 169-188, 2012.

Hägerstrand, T.: What about People in Regional Science?, Regional Science Association Papers, 24, 7-21, 1970.
Häußermann, H., Läpple, D., und Siebel, W.: Stadtpolitik, Suhrkamp Verlag, Frankfurt am Main, 2008.

Hilti, N.: Multilokales Wohnen - Bewegungen und Verortungen, Informationen zur Raumentwicklung, 1, 77-86, 2009.

Hofmeister, S.: Alles zu gleicher Zeit am gleichen Ort? Verdichtung von Raum und Zeit: das Ende der „Verinselung“, in: Zeitvielfalt. Wider das Diktat der Uhr, Herausgeber: Geißler, K. A., Kümmerer, K., und Sabelis, I., Hirzel, Stuttgart, 97-112, 2006.

Hörning, K. H., Gerhard, A., und Michailow, M.: Zeitpioniere. Flexible Arbeitszeiten, neuer Lebensstil, Suhrkamp Verlag Frankfurt am Main, 1998.

Ibert, O. und Thiel, J.: Situierte Analyse, dynamische Räumlichkeiten?, Z. Wirtsch., 53, 209-223, 2009.

Ibert, O., Pflanz, K., und Schmidt, S.: Spiel auf vielen Bühnen wie Musicaldarsteller ihre Vulnerabilität und Resilienz auf dem Arbeitsmarkt konstruieren, IRS Working Paper 46, Erkner, 2012.

Jürgens, K.: Arbeits- und Lebenskraft - Reproduktion als eigensinnige Grenzziehung, VS Verlag für Sozialwissenschaften, Wiesbaden, 2009.

Jurczyk, K., Schier, M., Szymenderski, P., Lange, A., und Voß, G. G.: Entgrenzte Arbeit - entgrenzte Familie, Grenzmanagement im Alltag als neue Herausforderung, Edition Sigma, Berlin, 2009.

Lange, B.: SWOT Analyse der Kreativwirtschaft in Leipzig, Arbeitspapier im Projekt Creative Cities (INTERREG IV B), Leipzig, 31-12-2010, 2010.

Läpple, D., Mückenberger, U., und Oßenbrügge, J.: Vorwort: die Gestaltung der Raum-Zeit-Muster ,postfordistischer" Stadtquartiere, in: Zeiten und Räume der Stadt, Herausgeber: Läpple, D., Mückenberger, U., und Oßenbrügger, J., Verlag Barbara Budrich, Opladen, 9-23, 2010.

Larsen, J., Urry, J., and Axhausen, K.: Mobilities, Networks, Geographies, Ashgate, 2006.

Lippuner, R.: Wissenschaft und Alltag, Zum theoretischen Problem Geographien der Praxis zu beobachten, Dissertation, Jena, 2003.

Oßenbrügge, J. und Vogelpohl, A.: Entgrenzte Zeiten - begrenzte Räume: Stadt(teil)entwicklung in raum-zeitlicher Perspektive, in: Zeiten und Räume der Stadt, Herausgeber: Läpple, D., Mückenberger, U., und Oßenbrügger, J., Verlag Barbara Budrich, Opladen, 65-88, 2010.

Oßenbrügge, J., Pohl, T., und Vogelpohl, A.: Entgrenzte Zeitregime und wirtschaftsräumliche Konzentrationen. Der Kreativsektor des Hamburger Schanzenviertels in zeitgeographischer Perspektive, Z. Wirtsch., 53, 249-263, 2009.

Pohl, T.: Entgrenzte Stadt: Räumliche Fragmentierung und zeitliche Flexibilisierung in der Spätmoderne, Transcript, Bielefeld, 2009.

Pohl, T.: Chronomaps als Repräsentations- und Kommunikationsmittel der raum-zeitlichen Strukturierung, in: Zeiten und Räume der Stadt, Herausgeber: Läpple, D., Mückenberger, U., und Oßenbrügge, J., Verlag Barbara Budrich, Opladen, 159-181, 2010.

Pütz, T.: Regionalisierung von Arbeiten und Wohnen, Statistischer Quartalsbericht 1/2010, 47-49, 2010.

Rosa, H.: Beschleunigung - Die Veränderung der Zeitstrukturen in der Moderne, Suhrkamp Verlag, Frankfurt am Main, 2005.

Scheiner, J.: Aktionsraumforschung auf phänomenologischer und handlungstheoretischer Grundlage, Geogr. Z., 86, 50-66, 1998.

Schiffbänker, H. und Papouschek, U.: Was ist überhaupt Arbeitszeit? Arbeitszeit und private Zeit in den Wiener „Creative 
Industries“, SWS Rundschau, 49, 84-105, 2008.

Schmitz, S.: Revolutionen der Erreichbarkeit, Gesellschaft, Raum und Verkehr im Wandel, Stadtforschung aktuell 83, Opladen, 2001.

SEKo: Integriertes Stadtentwicklungskonzept Stadt Leipzig 2020, Leipzig, 2009.

Thiel, J.: Lokale Dimensionen der Entgrenzung von Arbeit - ein arbeitsgeographischer Blick auf die Kultur- und Medienwirtschaft, Geogr. Z., 96, 62-77, 2008.

von Streit, A.: Entgrenzter Alltag - Arbeiten ohne Grenzen? Das Internet und die raum-zeitlichen Organisationsstrategien von Wissensarbeitern, Reihe „Gesellschaft der Unterschiede“ transcript, Bielefeld, 2011.
Weichhart, P.: Multilokalität - Konzepte, Theoriebezüge, Forschungsfragen, Informationen zur Raumentwicklung, 1, 1-14, 2009.

Wellman, B.: "Little boxes, glocalization, and networked individualism." Digital cities II: Computational and sociological approaches, 337-343, 2002.

Werlen, B.: Sozialgeographie alltäglicher Regionalisierungen Bd. 1: Zur Ontologie von Gesellschaft und Raum, Franz Steiner Verlag, Stuttgart, 1995.

Werlen, B.: Sozialgeographie, UTB, Stuttgart, 2000. 